University of Nebraska - Lincoln

DigitalCommons@University of Nebraska - Lincoln

6-11-2003

\title{
Theoretical study on the optical properties of polyvinylidene fluoride crystal
}

\author{
Chun-gang Duan \\ University of Nebraska at Omaha, cgduan@clpm.ecnu.edu.cn \\ Wai-Ning Mei \\ University of Nebraska at Omaha, physmei@unomaha.edu \\ Wei-Guo Yin \\ University of Nebraska-Omaha \\ Jianjun Liu \\ University of Nebraska - Lincoln, jliu2@unl.edu \\ J. R. Hardy \\ University of Nebraska - Lincoln
}

See next page for additional authors

Follow this and additional works at: https://digitalcommons.unl.edu/physicsducharme

Part of the Physics Commons

Duan, Chun-gang; Mei, Wai-Ning; Yin, Wei-Guo; Liu, Jianjun; Hardy, J. R.; Bai, Mengjun; and Ducharme, Stephen, "Theoretical study on the optical properties of polyvinylidene fluoride crystal" (2003). Stephen Ducharme Publications. 36.

https://digitalcommons.unl.edu/physicsducharme/36

This Article is brought to you for free and open access by the Research Papers in Physics and Astronomy at DigitalCommons@University of Nebraska - Lincoln. It has been accepted for inclusion in Stephen Ducharme Publications by an authorized administrator of DigitalCommons@University of Nebraska - Lincoln. 


\section{Authors}

Chun-gang Duan, Wai-Ning Mei, Wei-Guo Yin, Jianjun Liu, J. R. Hardy, Mengjun Bai, and Stephen Ducharme 


\title{
Theoretical study on the optical properties of polyvinylidene fluoride crystal
}

\author{
Chun-gang Duan ${ }^{1}$, W. N. Mei ${ }^{1}$, Wei-Guo Yin ${ }^{1}$, Jianjun Liu ${ }^{2}$, \\ J. R. Hardy ${ }^{2}$, Mengjun Bai ${ }^{2}$, and Stephen Ducharme ${ }^{2}$ \\ ${ }^{1}$ Department of Physics, University of Nebraska-Omaha, Omaha, NE 68182-0266, \\ USA \\ ${ }^{2}$ Department of Physics and Center for Materials Research and Analysis, University \\ of Nebraska-Lincoln, Lincoln, NE 68588, USA \\ Corresponding author: Chun-gang Duan — email dcg@cosmos.unomaha.edu
}

\begin{abstract}
We report our first-principles studies on the linear and non-linear optical properties of ferroelectric polyvinylidene fluoride crystal. Calculated values of the refractive indices agree well with experiments. As regards the second-harmonic-generation coefficients, theoretical values are larger than experimental ones. Possible reasons for the discrepancy are discussed.
\end{abstract}

Polyvinylidene fluoride (PVDF) and its copolymers with trifluoroethylene (TrFE) have been studied extensively due to their unique piezoelectric, pyroelectric, and ferroelectric properties [1-4]. They are widely used in industry as low-cost electromechanical devices such as transducers and detectors. Furthermore, the ferroelectric phases of PVDF copolymer crystals also have good non-linear optical (NLO) properties $[2,3]$. They have quite large optical windows (1200-200 nm), which makes them good candidates for use as frequency conversion materials working in the ultraviolet region. They can also be used in optical memory devices [5]. In addition, the NLO quality of this material can be significantly improved by hosting molecules of high NLO susceptibility in the amorphous region, which is a great advantage of polymers [6].

Experimentally, there are many reports on the determination of the optical constants of PVDF [7-10]. However, the crystalline polymers used in experiments often consist of crystalline regions comprising microcrystals and non-crystalline areas between the microcrystals [11], and the orientations of microcrystals in the samples used in experiments generally have a broad distribution. Thus those experimental values differ substantially from each other. As regards the theoretical side, to our knowledge, there has been only very limited investigation of the optical properties of thismaterial. Cakmak and Wang [12] and Spector and Stein [13] have given reports on the refractive indices of PVDF. However, their quantum chemistry calculations strongly depend on the choices of bond parameters, which results in a large uncer- 
tainty [12]. Thus it is necessary to carry out a first-principles study on the optical properties of single-crystal PVDF, which is a starting point for understanding the microscopic mechanism governing the optical behaviors of the complicated specimens used in experiments.

In a recent letter [14], we reported theoretical results on the energy band structure and band symmetries of PVDF. The calculated results agree well with photoemission experiments [15]. As a further step, in this paper,we report our first-principles calculation of the linear and NLO constants of the ferroelectric phase (the polar $\beta$-phase, form I) of PVDF.

The first-principles band structure approach applied in this work is the full-potential linear-augmented-plane-wave (FLAPW) method [16]. The exchange-correlation potential is treated in the generalized gradient approximation (GGA) [17] of density functional theory. The crystal structure and calculation details such as the cut-off energy, muffin-tin radii, and bandstructure, are presented in our previous work [14]. Here it is important to point out that the $b$-axis in our calculation is the polar axis, whereas the $c$-axis is the chain axis of PVDF. The energy band structure and density of states (DOS) of PVDF are presented in figure 1 for the convenience of later discussion.

In this work, we adopt the formalism of Hughes and Sipe [18] to calculate the optical susceptibilities. Their formula used in the calculation of SHG coefficients was rearranged by Rashkeev et al [19] to show Kleinman symmetry explicitly. Lin et al [20] improved the formula in the static limit by avoiding possible divergent terms; thus they were able to achieve good convergence on the $k$-point sampling, as we did before by using a different formula that does not present Kleinman symmetry explicitly [21]. Here we point out that the formula of Lin et al can be further simplified by abandoning the so-called two-bands term, which can be proved to be zero after some straightforward algebra. Specifically, the expression for $\chi^{(2)}$ in the static limit can be written as

$$
\begin{aligned}
\chi_{i j k}^{(2)}=\frac{e^{3}}{2 \hbar^{2} m^{3}} & \int_{B Z} \frac{\mathrm{d} k}{4 \pi^{3}} \mathrm{P}(i j k)\left[\sum_{v v^{\prime} c} \operatorname{Im}\left(p_{v^{\prime} v}^{i} p_{v c}^{j} p_{c v^{\prime}}^{k}\right)\left(\frac{1}{\omega_{c v}^{3} \omega_{c v^{\prime}}^{2}}+\frac{2}{\omega_{c v}^{4} \omega_{c v^{\prime}}}\right)\right. \\
& \left.+\sum_{v c c^{\prime}} \operatorname{Im}\left(p_{c v}^{i} p_{v c^{\prime}}^{j} p_{c^{\prime} c}^{k}\right)\left(\frac{1}{\omega_{c v}^{3} \omega_{c^{\prime} v}^{2}}+\frac{2}{\omega_{c v}^{4} \omega_{c^{\prime} v}}\right)\right],
\end{aligned}
$$

where $i, j, k$ are Cartesian components, $\mathrm{P}(i j k)$ denotes full permutation over $i, j, k$ and explicitly shows Kleinman symmetry of the SHG coefficients, $v\left(v^{\prime}\right)$ and $c\left(c^{\prime}\right)$ denote valence and conduction bands, respectively. $p_{m n}$ is the momentum matrix element connecting states $m$ and $n$ and the band energy difference is denoted as $\hbar \omega_{m n}$. Scissors operators are introduced to overcome the band gap underestimation problem [22]. In this calculation, the energy shift is chosen to be $0.6 \mathrm{eV}$ to fit the experimental band gap [14]. Since the calculated energy gap is very close to the experimental one in our case, there is no need to use other sophisticated techniques applied in theoretical studies of the optical properties of polymer materials such as polyacetylene [23]. Furthermore, the validity of the scissors approximation is strongly supported by recent SHG experiments on GaAs [24].

The linear optical properties of PVDF are investigated using 48 special $k$-points in the irreducible Brillouin zone. The results are very good on $k$-points convergence. The difference between the dielectric constants obtained with 12 and $48 k$-points is within $0.5 \%$. Since the system that we studied is aperfect biaxial crystal with orthorhombic structure, there are three different principal indices of refraction, i.e., $n_{a}, n_{b}$, and $n_{c}$, in which $a, b$, and $c$ represent the photon polarization directions along the crystal axes $a, b$, and $c$, respectively. Calculated values of the refractive indices are listed in Table 1, together with corresponding experimental and other theoretical values. In fact the formula used to calculate the refractive indices is well documented and ours is taken from [25]. As we can see from the table, the magnitudes of our calculated refractive indices agree well with observations. However, the agreement of 

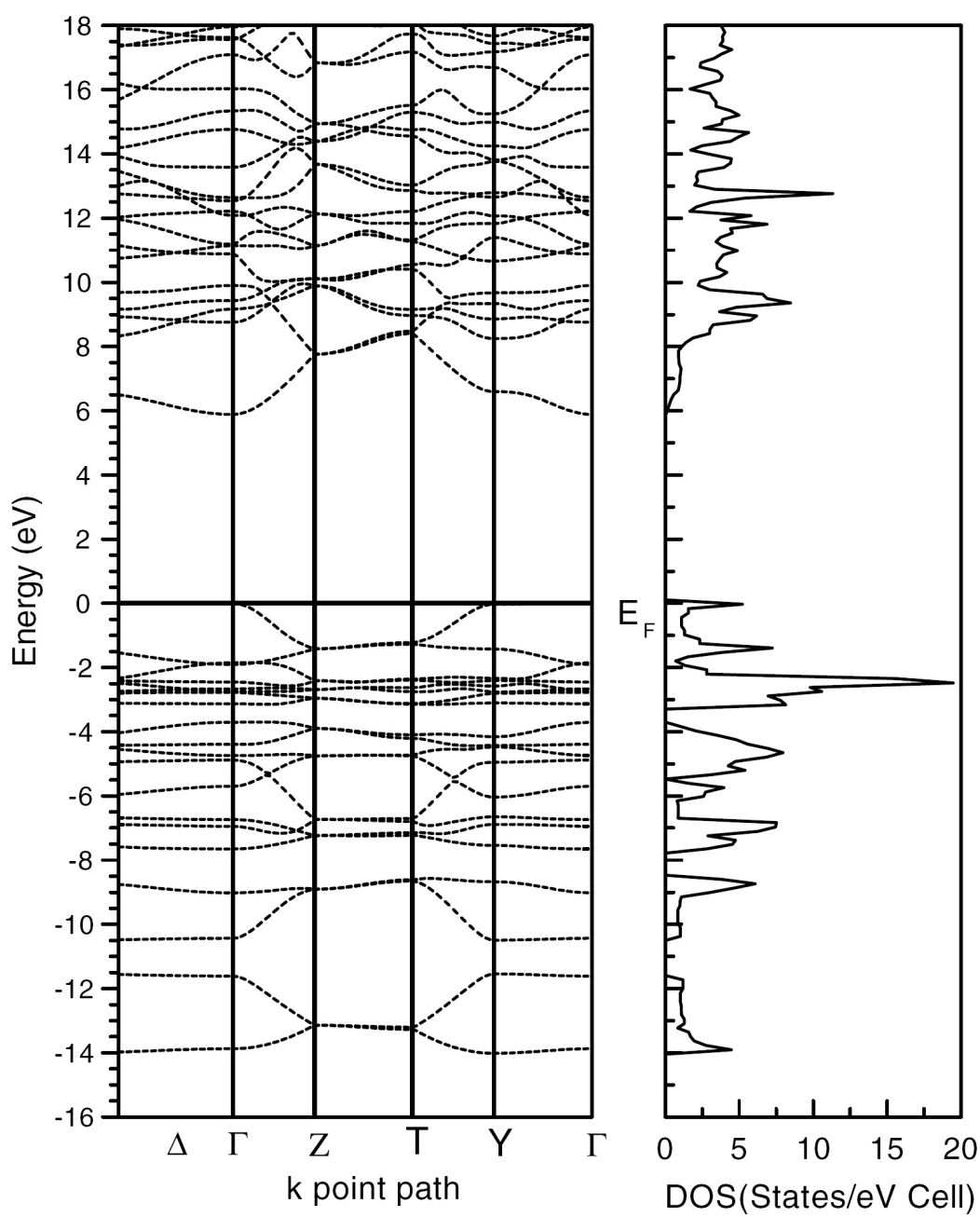

Figure 1. The calculated band structure and DOS of PVDF.

the anisotropic orders is not good. Our calculations found $n_{a}>n_{c}>n_{b}$, whereas experimental results show that $n_{c}$ has the largest value. The dispersions of the refractive indices are plotted in figure 2. Assuming that the wavelength of the incident light is $1.06 \mu \mathrm{m}$, we obtained the average dispersion as $n_{2 \omega}-n_{\omega}=0.0082$, very close to the experimental value 0.0077 [8]. We also noticed that $n_{c}$ becomes larger than $n_{a}$ after $4.5 \mathrm{eV}$.

Figure 3 shows the calculated linear absorption spectra for the three different polarization directions of PVDF. As can be seen from the figure, the anisotropic structure is quite obvious, i.e., the absorption of the direction $c$ (the chain direction) starts from about $6.5 \mathrm{eV}$, corresponding to the value of the energy gap, whereas the absorptions of the other two directions (perpendicular to the chain) start from almost $8 \mathrm{eV}$. As we can see from figure 1, the short terrace that starts at $6.5 \mathrm{eV}$ and extends about $2 \mathrm{eV}$ at the lower end of the conduction bands in the DOS plot is solely formed by the first conduction band, which mainly consists of C $2 \mathrm{p}$ electron states. A detailed oscillator strength analysis reveals that the first peak in the plot of the absorption coefficients corresponds to the transition from the highest occu- 


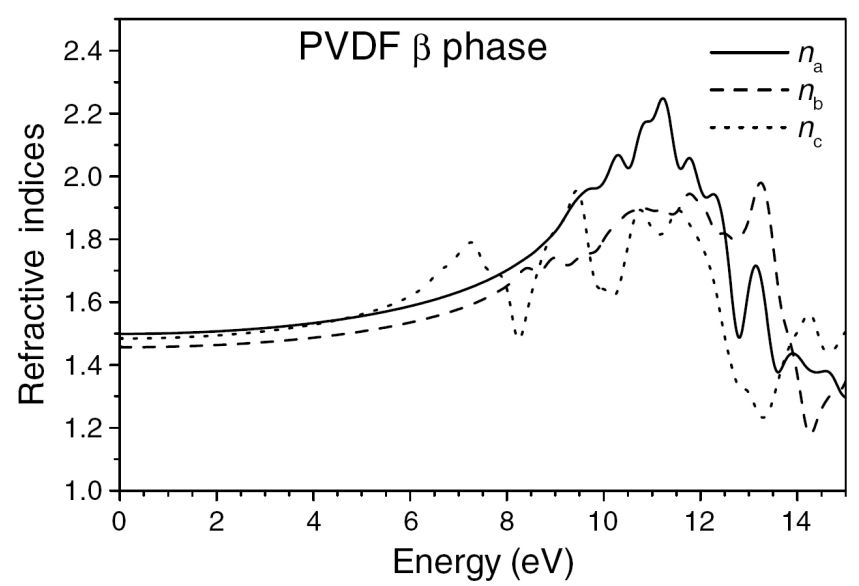

Figure 2. Calculated dispersion curves for the refractive indices of PVDF. Labels $a$, $b$, and $c$ represent the photon polarization directions along the crystal axes $a, b$, and $c$, respectively.

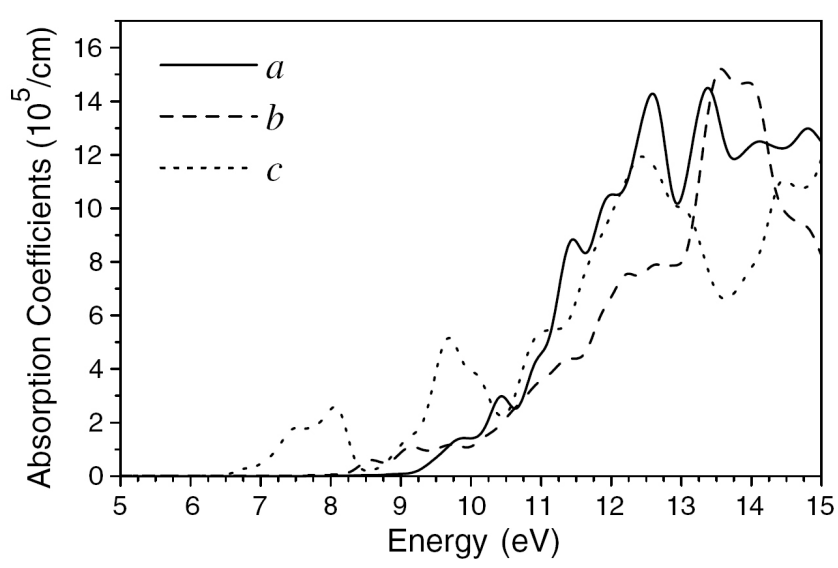

Figure 3. Linear absorption coefficients of PVDF.

Table 1. Refractive indices of PVDF crystal.

\begin{tabular}{lccc}
\hline & $n_{a}$ & $n_{b}$ & $n_{c}$ \\
\hline Theory & 1.499 & 1.456 & 1.484 \\
$\quad$ Current & 1.545 & 1.522 & 1.448 \\
Cakmak and Wang $^{\mathrm{a}}$ & 1.483 & 1.548 & 1.629 \\
$\quad$ Experiment & & & \\
Broussoux and Micheron $^{\mathrm{b}}$ & 1.436 & 1.425 & 1.444 \\
\hline
\end{tabular}

${ }^{a}$ Reference [12].

${ }^{\mathrm{b}}$ Reference [7].

pied molecular orbital in which F 2 p states are dominant to the lowest unoccupied molecular orbital. And only the transition along the chain direction is favourable. This again reveals the one-dimensional nature of the PVDF crystal. 


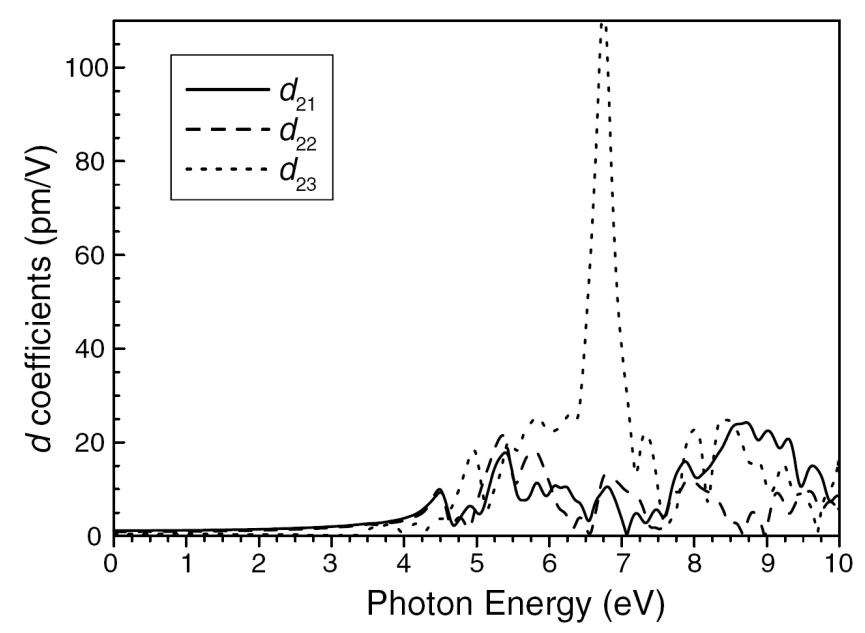

Figure 4. Calculated dispersion curves for the $d$-coefficients of PVDF.

Table 2. SHG coefficients $d_{21}, d_{22}, d_{23}$ for PVDF crystal, in units of $\mathrm{pm} \mathrm{V}^{-1}$.

\begin{tabular}{lccc}
\hline & $d_{21}$ & $d_{22}$ & $d_{23}$ \\
\hline Theory & 2.01 & 1.66 & 0.26 \\
Experiment $(\lambda=1060 \mathrm{~nm})$ & & & \\
$\quad$ Bergman et $^{\mathrm{a}} \mathrm{l}^{\mathrm{a}}$ & 0.23 & 0.47 & $\sim 0$ \\
Berge et $^{\mathrm{b}}$ & 0.05 & 0.22 & 0.05 \\
\hline
\end{tabular}

${ }^{\mathrm{a}}$ Reference [2].

${ }^{\mathrm{b}}$ Reference [8].

Then we calculated the SHG coefficients of the $\beta$-phase of PVDF. Since the point group of PVDF is $\mathrm{C}_{2 \mathrm{v}}(\mathrm{mm} 2)$ and the $\boldsymbol{b}$-axis is the twofold symmetry axis, there are three independent SHG components, i.e., $\left(=\frac{1}{2} \chi_{211}^{(2)}\right), d_{22}\left(=\frac{1}{2} \chi_{222}^{(2)}\right)$, and $d_{23}\left(=\frac{1}{2} \chi_{233}^{(2)}\right)$. The calculated results and available experimental values are listed in table 2 for comparison. As we can see from the table, the theoretical values are much larger than the experimental values. From the dispersion curves of the $d$-coefficients (see figure 4 ), we can see that the behaviors of the components $d_{21}$ and $d_{22}$ are quite similar. The component $d_{23}$ is rather small in the static limit, but it becomes quite large after $3.5 \mathrm{eV}$ and reaches its extremely large peak value at around $7 \mathrm{eV}$. However, SHG experiments in that energy range are rarely carried out, so data were scarce.

PVDF has important applications because of its electro-optical properties [7]. Using the relationship between the linear electro-optical (LEO) coefficients $r_{a b c}$ and the SHG coefficients $\chi_{a b c}$ in the static limit [18], i.e.,

$$
r_{a b c}=-\frac{2 \chi_{a b c}}{n_{a}^{4}},
$$

where $a, b, c$ are Cartesian components and $n_{a}$ is the index of refraction in the $a$-direction, we found the three independent LEO coefficients $r_{211}, r_{222}$, and $r_{233}$ to be $1.78,1.48$, and 0.23 $\mathrm{pm} \mathrm{V}^{-1}$, respectively. Again, these values are much larger than the experimental values 0.21 , 0.38 , and $0.10 \mathrm{pm} \mathrm{V}^{-1}[7]$.

We attribute these discrepancies, together with the mismatch of the theoretical and experimental anisotropic orders of the refractive indices, to the fact that the samples used in those experiments are not perfect single crystals, or not even single phase. For example, the 
chain axes of the specimens used by Berge et al [8] are randomly oriented in the plane of the film. Thus their SHG coefficients $d_{21}$ and $d_{23}$ are equal. In such cases, the refractive indices and SHG coefficients are actually averaged values. The orientation problem may not be very serious in the linear case, but in the determination of non-linear coefficients that are strongly orientation dependent, this would make a big difference.

Another important factor which causes a discrepancy between the theory and experiments is the copolymer effect. As is well known, the samples most widely used experimentally are actually PVDF copolymers (P(VDF-TrFE), 70:30). To study the copolymer effect, we calculated the optical constants of PTrFE, whose electronic structure has also been studied in our previous work [14]. We found that the average values of the refractive indices changed significantly from PVDF (1.48) to PTrFE (1.29). And the anisotropic order of PTrFE $\left(n_{c}>n_{a}>n_{b}\right)$ is also different from that of PVDF. Thus we were able to confirm that the replacement of hydrogen atoms with fluorine atoms will reduce the overall refractive index of the polymer, which agrees with the conclusion of Cakmak and Wang [12]. In the non-linear case, the copolymer effect is even more obvious, since PTrFE has a different point group to PVDF and thus different independent SHG components.

In summary, we have studied the linear and NLO properties of PVDF by means of a first-principles calculation. The values of the calculated refractive indices are in good agreement with experiments. But the anisotropic order of the refractive indices is different from the observations. We also calculated the SHG and LEO coefficients of this material. The agreement with experiments is not good. We attribute these discrepancies to the non-singlecrystal samples used in experiments and the copolymer effect. However, other factors such as temperature, and surface and local field corrections may also play important roles. Our calculations, unlike previous quantum chemistry analysis, give an unambiguous prediction of the optical properties of the ideal $\beta$-phase of PVDF, which might be helpful for determining the actual orientation of the molecular axis in films used in experiments.

\section{Acknowledgments}

This work was supported by the Nebraska Research Initiative, the Nebraska-EPSCoR-NSF Grant EPS-9720643 and the Department of the Army Grants DAAG 55-98-1-0273 and DAAG 55-99-1-0106. S.D. and W.N.M. are grateful for the support from the Office of Naval Research.

\section{References}

[1] Kawai H 1969 Japan. J. Appl. Phys. 8975

[2] Bergman J G, McFee J H, and Crane G R 1971 Appl. Phys. Lett. 18203

[3] McFee J H, Bergman J G, and Crane G R 1972 Ferroelectrics 3305

[4] Kepler R G and Anderson R A 1978 J. Appl. Phys. 491232

[5] Berry M H and Gookin D M 1988 Nonlinear Optical Properties of Organic Materials (Proc. SPIE vol 971) ed G Khanarian (Bellingham, WA: SPIE Optical Engineering Press) p 154

[6] Hill J R, Dunn P L, Davies G J, Oliver S N, Pantelis P, and Rush D J 1987 Electron. Lett. 23700

[7] Broussoux D and Micheron F 1980 J. Appl. Phys. 512020

[8] Berge B, Wicker A, Lajzerowicz J, and Legrand J F 1989 Europhys. Lett. 9657

[9] Krüger J K et al 1997 Phys. Rev. B 553497

[10] Tajitsu Y, Kato S, Okubo K, Ohigashi H, and Date M 2000 J. Mater. Sci. Lett. 19295

[11] Wang T T, Herbert J M, and Glass A M 1988 The Applications of Ferroelectric Polymers (Glasgow: Blackie)

[12] Cakmak M and Wang Y 1989 J. Appl. Polym. Sci. 37977

[13] Spector K S and Stein R S 1991 Macromolecules 242083

[14] Duan C G, Mei W N, Hardy J R, Ducharme S, Choi J, and Dowben P A 2003 Europhys. Lett. 61 5760 
[15] Choi Jaewu et al 2000 Phys. Rev. B 615760 and references

[16] Blaha P, Schwarz K, Madsen G K H, Kvasnicka D, and Luitz J 2001 WIEN2k, an Augmented Plane Wave + Local Orbitals Programme for Calculating Crystal Properties Technical University of Vienna, Austria (ISBN 3-9501031-1-2)

[17] Perdew J P, Burke K, and Ernzerhof M 1996 Phys. Rev. Lett. 773865

[18] Hughes J L P and Sipe J E 1996 Phys. Rev. B 5310751 and references

[19] Rashkeev S N, Lambrecht W R L, and Segall B 1998 Phys. Rev. B 579705

[20] Lin Jiao, Lee M, Liu Zhi-Ping, Chen Chuangtian, and Pickard C J 1999 Phys. Rev. B 6013380

[21] Duan CG, Li J, Gu Z Q, and Wang D S 1999 Phys. Rev. B 59 369; Duan C G, Li J, Gu Z Q, and Wang D S 1999 Phys. Rev. B 609435

[22] Levine Z H and Allan D C 1991 Phys. Rev. Lett. 66 41; Levine Z H and Allan D C 1991 Phys. Rev. B 4412781

[23] Puschnig P and Ambrosch-Draxl C 2002 Phys. Rev. Lett. 89056405

[24] Bergfeld S and Daum W 2003 Phys. Rev. Lett. 90036801

[25] Li J, Duan C G, Gu Z Q, and Wang D S 1998 Phys. Rev. B 572222 